\title{
Síndrome de dolor regional complejo en niños: posibilidades de tratamiento
}

\author{
M. J. Rodríguez-López, M. Fernández-Baena y J. A. Yáñez-Santos \\ Unidad de Tratamiento del Dolor. Hospital Regional Universitario "Carlos Haya”. Málaga
}

Rodríguez-López MJ, Fernández-Baena M, Yáñez-Santos JA. Síndrome de dolor regional complejo en niños: posibilidades de tratamiento. Rev Soc Esp Dolor 2014; 21(3): 138-145.

\begin{abstract}
Background: The IASP defined complex regional pain syndrome (CRPS) as "a variety of painful conditions of regional location alter a injury with distal predominance of abnormal symptoms, exceeding in magnitude and duration the expected clinical course of the initial incident, often rausing significant motor impairment, with variable progression over time".

CRPS is characterized by severe pain, accompanied by other symptoms among which allodynia. It is a well-defined clinical entity in adults, but until recently lie doubted his presence in children and adolescents, today fully accepted this fact.

Presents some differences with adult CRPS, including that is more common in girls and affects mainly the lower extremities.

Case reports: Nine children (five boys and four girls) aged between 8 and 13 years old diagnosed with CRPS, the trigger box has been in most patients for the presence of mild trauma. All patients are subject to the same treatment algorithm: a) Drug tretment and rehabilitative therapy associated with the application of the capsaicin patch $8 \%$; and b) interventional techniques (epidural infusion of local anesthetic and/or electrical stimulation spinal cord) in patients whose relief is not adequate.

The complete remission was obtained in nine patients using the treatment algorithm.
\end{abstract}

Discussion: The diagnosis of CRPS should be done as early as possible which will allow initiation of treatment also is.
While most children will respond to conventional treatment, there are cases where they are given the use of interventional techniques.

Key words: Complex regional pain syndrome (CRPS). Children. Chronic pain. Pain treatment. Interventional treatment.

\section{RESUMEN}

Introducción: La IASP define el síndrome de dolor regional complejo (SDRC) como "una variedad de condiciones dolorosas de localización regional, posterior a una lesión, que presentan predominio distal de síntomas anormales, excediendo en magnitud y duración al curso clínico esperado del incidente inicial, ocasionando con frecuencia un deterioro motor importante, con una progresión variable en el tiempo".

El SDRC se caracteriza por dolor intenso, acompañado de otros síntomas entre los que destaca la alodinia. Es una entidad clínica muy bien definida en los adultos, pero hasta hace poco tiempo se dudaba de su presencia en niños y adolescentes, hoy este hecho se acepta plenamente. Presenta algunas diferencias con el SDRC del adulto, entre ellas que es más frecuente en niñas y que afecta fundamentalmente a las extremidades inferiores.

Casos clínicos: Nueve niños (cinco niños y cuatro niñas) con edades comprendidas entre los 8 y los 13 años de edad diagnosticados de SDRC; el desencadenante del cuadro ha sido en la mayoría de los pacientes la presencia de un traumatismo de intensidad leve. A todos los pacientes se les aplicó el mismo algoritmo de tratamiento: a) tratamiento farmacológico más terapia rehabilitadora asociado a la aplicación del parche de capsaicina al $8 \%$; y b) técnicas intervencionistas (infusión epidural de anestesia local y/o estimulación eléctrica medular) en aquellos pacientes cuyo alivio no es el adecuado.

La remisión obtenida ha sido completa en los nueve pacientes con la utilización del algoritmo de tratamiento.

Discusión: El diagnóstico del SDRC debe hacerse lo más precoz posible lo que permitirá que el inicio del tratamiento también lo sea. Si bien la mayoría de los niños van a responder 
al tratamiento convencional, existen casos donde se encuentran indicadas la utilización de las técnicas intervencionistas.

Palabras clave: Síndrome de dolor regional complejo. Niños. Dolor crónico. Tratamiento convencional. Tratamiento intervencionista.

\section{INTRODUCCIÓN}

La IASP (Asociación Internacional para el Estudio del Dolor) define el síndrome de dolor regional complejo (SDRC) como: "variedad de condiciones dolorosas de localización regional, posterior a una lesión, que presentan predominio distal de síntomas anormales, excediendo en magnitud y duración al curso clínico esperado del incidente inicial, ocasionando con frecuencia un deterioro motor importante, con una progresión variable en el tiempo" (1).

El cuadro clínico incluye: dolor de intensidad severa, alodinia y/o hiperalgesia, edema de la extremidad afectada acompañado de cambios en la temperatura y en la coloración de la extremidad, con aparición de cambios tróficos tanto en la coloración como en la textura de la piel, asociado con un mayor crecimiento tanto del pelo como de las uñas.

El SDRC es una entidad clínica muy bien definida en los adultos, si bien durante mucho tiempo se puso en duda su presencia en niños y adolescentes. Esta situación ha ido evolucionando en estos últimos años debida, sobre todo, a la publicación de diversos artículos en los cuales se ha descrito claramente la existencia de este síndrome tanto en niños como en adolescentes.

Estudios recientes (2-4) han demostrado que, a diferencia de la población adulta, el $90 \%$ de los casos reportados en niños aparecen en niñas, con un rango de edad comprendido entre los 8 y los 16 años y que se afectan más frecuentemente las extremidades inferiores, especialmente los pies. El SDRC en los niños se caracteriza, por lo general, por un cuadro de dolor con una intensidad severa, acompañado de alodinia, sobre todo táctil y con signos de disautonomía. En la mayoría de los casos existe la presencia de un traumatismo previo de una intensidad menor, si bien existen casos en los cuales no hay ninguna causa razonable que pueda ser identificada.

El dolor suele ser intenso, con características quemantes, asociado a cambios tanto en la temperatura como en la coloración de la extremidad afectada. La aparición de estos cambios indica la participación del simpático en su evolución; esta participación puede demostrarse con facilidad mediante la realización de un bloqueo del simpático (5).

Existe una gran variación en la respuesta al tratamiento por parte de los niños que lo padecen; esta respuesta variable se debe, probablemente, a una falta de compresión tanto de la etiología como de la fisiopatología del síndrome.
Este síndrome fue descrito por primera vez en el año 1766 por Hunter, si bien no es hasta 1864, durante la guerra de secesión norteamericana, cuando Silas W. Mitchell y colaboradores identificaron un cuadro de dolor severo que, acompañado de una serie de signos y síntomas específicos, se presentaba en soldados con heridas penetrantes producidas por arma de fuego o bien con lesiones de nervios periféricos secundarias a heridas por arma blanca; para describirlo en su libro sobre heridas de guerra, Mitchell usó el término causalgia (del griego kausas: calor y algos: dolor); con este describía un cuadro de dolor intenso con características quemantes en una o en varias extremidades, asociado a otros síntomas y signos y posterior a lesiones penetrantes, siendo esta descripción probablemente la primera que se hizo de este síndrome (6-8).

En 1900, Paul Sudeck, cirujano de Hamburgo, identifica el cuadro que lleva su nombre y lo describe como "atrofia ósea inflamatoria aguda" (9). En 1923, Leriche relaciona los trastornos vasomotores con una afectación del sistema simpático, describiendo un paciente aquejado de un cuadro de dolor severo secundario a una lesión de un nervio periférico, haciendo una descripción detallada de la "causalgia" describiéndolo como "un dolor urente, que empeoraba con estímulos simples y atípicos, con intensidad variable, que se aliviaba con el frío local y que, a largo plazo, se producían cambios atróficos cutáneos y musculares" (10).

En 1940, Homans lo denominó "causalgia minor" y Jonson describe en 1943, la relación entre el infarto de miocardio y los trastornos distróficos dolorosos en la extremidad izquierda. En 1946, Evans trató exitosamente a pacientes con dolor mediante la realización de bloqueos anestésicos e introdujo el término de "distrofia simpático refleja" (11). En 1947 Steinbrocker describió el "síndrome hombro-mano", señalando su frecuencia tras accidentes coronarios (12).

A partir de ese momento, este cuadro ha recibido distintos nombres, aplicándose por regla general, el término "algodistrofia" en la bibliografía europea y el de "distrofia simpático-refleja" en la literatura anglosajona. En 1953, Bonica en un intento de ordenar los criterios diagnósticos, acuñó el término de "distrofia simpático-refleja", describiendo los estados evolutivos de la distrofia y proponiendo para su tratamiento la aplicación de bloqueos nerviosos, periféricos y centrales (13).

En 1993, el Special Consensus Workshop de la IASP decidió unificar la denominación usando el término de "síndrome de dolor regional complejo". En 1994, el Comité de la IASP aceptó los criterios diagnósticos que han pasado a ser estrictamente clínicos (14).

El pronóstico del SDRC en niños guarda una relación directa con la rapidez del diagnóstico, de ahí la gran importancia que presenta el diagnóstico precoz del síndrome pues permite que el tratamiento se inicie lo más pronto posible $(2,15)$. Los dos pilares principales en los que se basa el tratamiento son: por una parte el control farmacológico 
del dolor y por otra la rehabilitación del miembro afectado (16). Sólo en aquellos pacientes en los que el dolor persiste a pesar del tratamiento sin que, al mismo tiempo, se consiga una mejoría en la función física de la extremidad afectada son los que deben considerarse candidatos a la utilización de técnicas intervencionistas.

El diagnóstico del SDRC se hace, en la mayoría de los casos, a partir de la historia clínica detallada del paciente; esta debe abarcar tanto una descripción detallada de los síntomas como de los signos clínicos acompañantes que, a su vez, se caracterizan por una notable variabilidad individual $(17,18)$.

Su diagnóstico clínico se debe basar en los criterios de la $\operatorname{IASP}(14)$ :

- Presencia, en la mayoría de las veces, de un traumatismo inicial.

- Presencia de la tríada: dolor, alodinia e hiperalgesia. La intensidad del dolor suele no guardar relación con la intensidad del traumatismo inicial desencadenante.

- Presencia de edema en la extremidad afectada, con cambios asociados en el flujo sanguíneo y con la presencia de una actividad sudomotora anormal.

- El diagnóstico clínico se hace cuando los síntomas indicados están presentes, teniendo siempre en cuenta, sobre todo, tanto la severidad del dolor como el grado de disfunción física.

\section{CASOS CLÍNICOS}

Presentamos un total de nueve niños tratados en la Unidad del Dolor de nuestro hospital entre julio de 2010 y julio de 2013, con edades comprendidas entre los 8 y los 13 años de edad (en el momento del inicio del tratamiento) diagnosticados de un SDRC, caracterizado por: presencia de dolor severo en una o en dos extremidades, acompañado de una serie de síntomas, entre los que destacan; alodinia intensa, edema de la articulación/es afectadas, impotencia funcional y presencia de alteraciones vasculares y/o cutáneas. En la mayoría de los pacientes el desencadenante del cuadro ha sido un traumatismo de intensidad leve ocurrido (en todos los casos) unos meses antes de su llegada a la Unidad.

De los nueve pacientes, cinco eran niños y cuatro niñas. Todos ellos presentaban dolor e impotencia funcional en una o ambas extremidades inferiores, existiendo el precedente de un traumatismo leve en ocho de ellos, siendo el más frecuente el esguince de tobillo; en siete pacientes el esguince se había producido en la extremidad afectada y en uno en la extremidad contra-lateral a la afectada.

Todos los pacientes fueron remitidos a la Unidad del Dolor por los Servicios de Traumatología o Reumatología Pediátrica, con un tiempo de evolución del SDRC comprendido entre los 2 meses a los 4 años. El motivo para su derivación fue, en todos los casos, dolor con intensidad severa que no había cedido con los tratamientos farmaco- lógicos previos (la mayoría de las veces: AINE y paracetamol) que se acompañaba de otros síntomas, tales como; alodinia táctil, edema del tobillo afectado, trastornos vasomotores y rigidez articular que impedía la realización de las maniobras de rehabilitación.

A la exploración, en todos los casos existía un cuadro de dolor continuo, intenso, quemante en el tercio inferior de la pierna, tobillo, talón y dorso del pie afectado que se exacerbaba ante el mínimo roce o movimiento, acompañado de: un gran edema articular del tobillo y dorso del pie, un cuadro de alodinia severa que a su vez dificultaba la exploración, cambios en la coloración del pie y del tobillo, cambios tróficos en la piel con alteraciones en el crecimiento del pelo y de las uñas, sudoración excesiva en la planta del pie y frialdad de la extremidad afectada. Todo ello motivó la presencia de un cuadro de impotencia funcional severa que impedía la movilidad de la extremidad afectada.

\section{Caso clínico 1}

Niña de 11 años de edad cuando se realizó el diagnóstico de esguince de tobillo izquierdo tratado en Traumatología Pediátrica. Fue diagnosticada de SDRC de tobillo y pie izquierdo por Reumatología Pediátrica e inicia tratamiento farmacológico; al no obtener alivio es derivada a la Unidad del Dolor.

Primero fue tratada farmacológicamente sin conseguir un alivio adecuado del dolor, por lo que se decidió (previa información y consentimiento paterno) implantar, en quirófano y bajo anestesia general, un catéter epidural lumbar iniciándose, a través de él, una infusión continua de anestesia local durante dos semanas. En ese tiempo el alivio del dolor fue total, pero este cesó al detener la administración de anestesia local. Por este motivo se decidió (previa información y consentimiento de los padres) la implantación, en quirófano y bajo anestesia general, de un electrodo octopolar en cordones medulares posteriores para estimulación de la pierna izquierda. El sistema se programó una vez despierta la paciente consiguiéndose una muy buena localización de las parestesias. Dos semanas más tarde se revisó a la paciente que continuaba con la buena respuesta tanto del dolor como del resto de la sintomatología, por lo que se decidió la implantación de un generador de impulsos implantable y programable y su conexión con el electrodo. En la actualidad continúa en tratamiento con la estimulación medular con remisión total del cuadro.

\section{Caso clínico 2}

Niña de 13 años de edad cuando se diagnosticó de SDRC de extremidad inferior derecha secundario a una fractura de tobillo. 
Fue derivada a la Unidad del Dolor por el Servicio de Traumatología Pediátrica. Se inició tratamiento farmacológico sin resultado analgésico adecuado por lo que se decidió (previa información y obtención del consentimiento por parte de los padres) la implantación, en quirófano y bajo anestesia general, de un catéter epidural lumbar conectado a una bomba externa para administrar una infusión continua de bupivacaína durante dos semanas. Se obtuvo un alivio completo del dolor así como del resto de los síntomas, pero este desapareció una vez retirada la infusión de bupivacaína volviendo a aparecer tanto el dolor como el resto de los síntomas acompañantes.

Previa información y consentimiento paterno se implantó en quirófano, bajo anestesia general, un electrodo octopolar en cordones medulares posteriores a nivel de T12-T9 y se conectó a un generador de impulsos eléctricos externo. Se programó una vez que la paciente estaba despierta consiguiéndose un buen nivel de parestesias con alivio completo del dolor. Ello permitió que la paciente iniciara la rehabilitación de la extremidad afectada. A las dos semanas se revisó observándose que continuaba el alivio sin que la paciente utilizase el generador de impulsos externo. Ante esta situación se desconectó el generador externo y se continuó con el tratamiento rehabilitador citando a la paciente cuatro semanas más tarde. Ante la persistencia de la ausencia de dolor y la mejoría de los síntomas acompañantes, se decidió (de acuerdo con los padres) explantar el electrodo medular. La paciente continúa con una remisión completa de los síntomas.

\section{Caso clínico 3}

Niño de 13 años de edad cuando sufrió un esguince de tobillo derecho. Fue diagnosticado y tratado por el Servicio de Traumatología Pediátrica y remitido a la unidad a los 6 meses del inicio del cuadro.

Se inició un tratamiento farmacológico (gabapentina más tramadol) con remisión completa del cuadro de dolor que permitió el inicio de la rehabilitación del pie. Continúa en igual situación.

\section{Caso clínico 4}

Niña de 8 años de edad cuando sufre un esguince del tobillo izquierdo. Se diagnostica de SDRC del tobillo y pie izquierdo que remite completamente en un par de semanas con tratamiento médico.

Dos meses después, y sin ningún traumatismo previo, se inicia un cuadro de dolor en el tobillo y el pie derecho que también se diagnostica de SDRC. Es derivada a la Unidad del Dolor por el Servicio de Traumatología Pediátrica a los dos meses de este diagnóstico.
Se inicia tratamiento con pregabalina más tramadol, más la aplicación del parche de capsaicina al $8 \%$ debido a la alodinia severa que presentaba, sin obtener un alivio adecuado ni del dolor ni del resto de la sintomatología acompañante.

Por ello se decide (previo consentimiento paterno) la implantación, en quirófano y bajo anestesia general, de un catéter epidural lumbar y su conexión a una bomba externa para la administración de una infusión continua de bupivacaína durante dos semanas; pasado ese tiempo se detiene la infusión y se retira el catéter. Remisión completa de los síntomas que continúa.

\section{Caso clínico 5}

Niña de 13 años de edad cuando se realiza el diagnóstico de SDRC en ambas extremidades inferiores sin traumatismo previo.

Derivada a la Unidad del Dolor por el Servicio de Traumatología Pediátrica a los cuatro meses del diagnóstico. Se administró tratamiento farmacológico más aplicación del parche de capsaicina al $8 \%$ con remisión completa del cuadro.

\section{Caso clínico 6}

Niño de 10 años de edad cuando se realiza el diagnóstico de SDRC. Esguince previo en el tobillo derecho con aparición de un cuadro de SDRC en el tobillo y pie derecho. Derivado a la Unidad a los cinco meses del diagnóstico.

Se le administra tratamiento farmacológico más aplicación del parche de capsaicina al 8 \% con remisión completa de los síntomas.

\section{Caso clínico 7}

Niña de 10 años de edad cuando se inicia el cuadro. Presenta esguince de tobillo izquierdo con desarrollo de un cuadro de SDRC de tobillo y pie izquierdo. Es remitida a la Unidad del Dolor por el Servicio de Reumatología Pediátrica a los cuatro años del diagnóstico.

Se le administra tratamiento farmacológico más aplicación de parche de capsaicina al $8 \%$ sin remisión del cuadro. Colocación en quirófano, bajo anestesia general y previo consentimiento paterno, de un catéter epidural lumbar y conexión a una bomba externa para administración de una infusión continúa de bupivacaína durante dos semanas. Retirada del catéter pasado ese tiempo. Remisión completa de los síntomas. 


\section{Caso clínico 8}

Niño de 11 años de edad cuando se realizó el diagnóstico. Presentó un traumatismo previo con esguince de tobillo izquierdo. Fue remitido a la Unidad 4 meses después del diagnóstico.

Se le administra tratamiento farmacológico más aplicación del parche de capsaicina al $8 \%$. Remisión completa de los síntomas.

\section{Caso clínico 9}

Niño de 9 años cuando se realizó el diagnóstico. Tenía traumatismo previo (esguince) de tobillo izquierdo. Fue remitido a la Unidad a los dos años del diagnóstico.

Se le administra tratamiento farmacológico más aplicación del parche de capsaicina. Alivio de la alodinia pero no del dolor profundo, por lo que se coloca en quirófano un catéter epidural conectado a una bomba externa para la administración durante dos semanas de un flujo continuo de bupivacaína. Retirada del catéter a las dos semanas con remisión completa de los síntomas.

\section{RESULTADOS}

En todos los pacientes el algoritmo de tratamiento aplicado ha sido el siguiente:

1. Tratamiento farmacológico mediante la administración de un anticonvulsivante gabapentinoide (gabapentina o pregabalina) asociado, en la mayoría de los casos, con un analgésico (preferentemente tramadol).

2. En los pacientes que además de dolor presentaban alodinia intensa y/o hiperalgesia, se asoció el tratamiento farmacológico con la aplicación del parche de capsaicina al $8 \%$. Se optó por esta asociación a partir de diciembre de 2011, previa información a los padres y consentimiento por escrito de ellos.

3. Cuando el alivio obtenido no ha sido suficiente para que el niño reinicie el tratamiento fisioterápico, se inicia la utilización de técnicas invasivas con la implantación, en quirófano, de un catéter epidural lumbar tunelizado y conectado a una bomba externa de infusión, que permita la infusión continua de bupivacaína durante un periodo de dos semanas.

4. Pasado ese tiempo, se retira la infusión epidural así como el catéter y se revisa el paciente a la semana. El dolor ha reaparecido en dos pacientes por lo que en estos casos se decidió la implantación, siempre en quirófano y bajo anestesia general, de un estimulador de cordones medulares posteriores.

Con la aplicación de este algoritmo de tratamiento la remisión ha sido completa en los nueve niños, obteniendo los siguientes resultados:

- Un niño remitió el cuadro de dolor y síntomas acompañantes sólo con la aplicación del tratamiento farmacológico más tratamiento rehabilitador.

- En tres niños se ha conseguido la remisión del cuadro mediante la asociación de tratamiento farmacológico y la aplicación de parche de capsaicina al $8 \%$ que permitió iniciar el tratamiento rehabilitador.

- En otros tres niños no fue posible iniciar el tratamiento rehabilitador a pesar de la aplicación del escalón anterior (tratamiento farmacológico más aplicación del parche de capsaicina al $8 \%$ ). En estos casos fue necesaria la implantación en quirófano de un catéter epidural lumbar y la administración, a su través, de una infusión continua de bupivacaína. En los tres niños la remisión conseguida ha sido completa.

- En dos niños, primeros casos de SDRC tratados en la Unidad, se utilizó el algoritmo de tratamiento, expuesto previamente, sin la aplicación del parche de capsaicina. En estos dos casos una vez explantado el catéter epidural reapareció el dolor y el resto de los síntomas por lo que se decidió la implantación de un electrodo para estimular los cordones medulares posteriores.

\section{DISCUSIÓN}

El término SDRC sirve para describir una variedad de cuadros de dolor crónico que, se cree, es secundario a una disfunción del SNC o periférico (19-21). Incluyen cambios en la coloración y la temperatura de la extremidad o región del cuerpo afectada, asociado a dolor intenso que suele ser desproporcionado a la lesión responsable de él $(14,22)$, alodinia hiperalgesia, trastornos vasomotores y sudomotores $(23,24)$.

El SDRC presenta un curso progresivo, caracterizado por dolor e impotencia funcional que da lugar al desarrollo de un círculo vicioso con aumento del dolor y la falta de uso de la/s extremidad/es afectada/s (25). Los signos de presentación más frecuentes son disminución en la amplitud de movimiento y alodinia en el $100 \%$ de los niños, cambios en la coloración de la piel en un $90 \%$ y cambios en la temperatura y edema en el $85 \%$. Para evitar la cronificación de esta situación es fundamental que tanto el diagnóstico como el tratamiento sean lo más precoces posible con el fin de conseguir una recuperación plena. Los niños en los que se diagnostican precozmente (menos de 3 meses) se logra una remisión completa, en comparación con aquellos cuyo diagnóstico se retrasa (10,6 vs. 21,5 semanas) (26).

Su retraso puede dar lugar a un cuadro de dolor prolongado, con deterioro funcional, junto con otras com- 
plicaciones que pueden originar a discapacidades de por vida. Junto a ello pueden existir trastornos psicológicos asociados como resultado del dolor, la falta de sueño y las limitaciones en la actividad (27).

El SDRC puede progresar a través de distintas etapas (temprana, distrófica y atrófica) (28-31). La existencia de estas etapas implica la presencia de un periodo crítico para orientar el tratamiento de una forma eficaz para conseguir la recuperación funcional de la extremidad afectada (32). En los niños, a menudo, se produce un retraso tanto en el diagnóstico como en el tratamiento debido a que el SDRC en ellos es relativamente raro y todavía no se conoce bien (25).

Existen una serie de características clínicas del SDRC en la población pediátrica que difieren del SDRC desarrollado en el adulto, tanto en su ubicación como en el género y el tratamiento:

- Las extremidades que se afectan con mayor frecuencia en los niños son las inferiores frente a las extremidades superiores que son las más afectadas en los adultos, en una proporción de 6:1, este hecho se debe, probablemente, a que las lesiones en los niños se deben, más frecuentemente, a los deportes y a los juegos y no al trabajo o al uso excesivo de la extremidad tal como ocurre en los adultos $(2,25,33,34)$. En el trabajo de Tan (35) la extremidad superior estaba afectada en el 23,3\% de los niños frente al 72,6 \% de las extremidades inferiores, siendo la causa más frecuente, el esguince de tobillo.

En todos los niños del presente artículo estaban afectadas las extremidades inferiores; esta afectación era unilateral en ocho casos y bilateral en uno de ellos. El SDRC se presentó después de un traumatismo en ocho casos.

- En los trabajos publicados el diagnóstico de SDRC aparece con mayor frecuencia en niñas que en niños, con una relación de 7:1 (5,26,35); por el contrario en los adultos esta relación entre mujeres y hombres es más baja (2:1).

En nuestro trabajo, esta proporción no aparece, ya que cinco de los pacientes son niños frente a cuatro niñas.

- Por último, la recuperación completa de la extremidad afectada es más frecuente en niños, mientras que la discapacidad a largo plazo es relativamente común en los adultos $(2,3,5,26)$.

Tanto el dolor como el resto de los signos y síntomas acompañantes han remitido en los nueve pacientes motivo de este artículo, si bien se ha conseguido aplicando distintas fases del algoritmo de tratamiento: a) con la aplicación del tratamiento farmacológico y la terapia física se ha obtenido éxito en un caso; b) en tres pacientes se ha conseguido la remisión mediante la combinación de tratamiento farmacológico más la aplicación del parche de capsaicina al $8 \%$; c) en otros tres pacientes para obtener la remisión ha sido necesario, además del tratamiento anterior (parche de capsaicina incluido), asociarle la implantación (en quirófano y bajo anestesia general) de un catéter epidural lumbar y el mantenimiento de una infusión continua de anestésico local durante un tiempo limitado (dos semanas); y d) en los dos pacientes restantes para obtener la remisión del cuadro y debido a la gravedad de sus síntomas, ha sido necesario además de la utilización de todo el algoritmo de tratamiento (menos la aplicación del parche de capsaicina al $8 \%$ ), la implantación de un electrodo en cordones medulares posteriores para estimulación eléctrica.

En el SDRC en niños para obtener un buen resultado analgésico es indispensable su reconocimiento y tratamiento precoz, debiendo utilizar para ello los criterios diagnósticos de la IASP (14), así como la aplicación inmediata de un algoritmo de tratamiento en el cual se contemple el tratamiento farmacológico, asociado a la fisioterapia y a la terapia conductual. En los trabajos de Lee, Sherry y Wilder, se comprueba cómo la movilización temprana asistida de apoyo conductual puede proporcionar un reversión dramática de los síntomas y signos $(16,36,37)$.

La rápida respuesta a estas medidas subraya la importancia que tiene realizar a tiempo el diagnóstico así como el inicio rápido del tratamiento, ya que en los pacientes que así se hace se obtiene una resolución completa de los síntomas. En los niños en los que el diagnóstico se hace precozmente (menos de 3 meses) se logra una remisión de los síntomas, en comparación con aquellos niños en los que el diagnóstico se retrasa (2-4).

El tratamiento inicial del SDRC se dirige a conseguir alivio del dolor y una normalización de la movilidad de la extremidad afectada; esto se logra mediante una combinación de fármacos junto con terapia física. Los fármacos más utilizados en el tratamiento del SDRC son: paracetamol, AINE, tramadol, antidepresivos tricíclicos o duales y anticonvulsivantes gabapentinoides $(3,26)$. Con la aplicación de este escalón de tratamiento se ha logrado la remisión de los síntomas en uno de los nueve pacientes.

Existen pacientes que presentan, asociado al dolor intenso, un cuadro de alodinia severa lo que va a dificultad que el paciente pueda iniciar y mantener un programa de rehabilitación física efectiva. Este cuadro de alodinia se ha tratado, en seis de los pacientes, combinando el tratamiento farmacológico con la utilización de la vía tópica, para ello se ha recurrido a la aplicación del parche de capsaicina al $8 \%$. Puesto que la utilización de dicho parche de capsaicina al $8 \%$ no está indicado en niños según su ficha técnica ha sido necesaria dar una información detallada a los padres del paciente de sus opciones terapéuticas, así como de las posibles complicaciones secundarias a su utilización y obtener el consentimiento paterno por escrito previo a la utilización del parche. Con su aplicación se ha conseguido una remisión completa del dolor y de la alodinia acompañante en tres de los seis pacientes (50\% de los casos), no 
aparecieron complicaciones secundarias a su aplicación en ninguno de los seis pacientes.

Cuando, con la aplicación del algoritmo de tratamiento expuesto anteriormente, no se consigue una remisión del dolor y del resto de la sintomatología acompañante es cuando están indicadas las distintas técnicas intervencionistas necesarias para facilitar la remisión del cuadro (37). Si bien antes de recurrir a ellas es necesario que se hayan agotado las distintas posibilidades de utilización de los tratamiento convencionales (38). Cuando estas técnicas no se aplican en los pacientes con síntomas graves, no sólo se pone en peligro la recuperación del paciente sino también su confianza en los médicos. Estos niños pueden desarrollar cambios irreversibles que involucran su funcionalidad, así como otras manifestaciones crónicas en la extremidad afectada tales como neuropatías periféricas y/o vasculopatías.

En esta serie de pacientes fue necesario recurrir a la utilización de distintas técnicas invasivas en cinco de los nueve niños $(55,5 \%)$, debido sobre todo a la continuación y progresión de los síntomas a pesar de la utilización del tratamiento conservador. En los cinco niños se implantó en quirófano y bajo anestesia general de un catéter epidural lumbar (previo consentimiento informado paterno), se tunelizó y se conectó a un sistema de infusión continua externo, a su través se inició la administración de una infusión continua de anestesia local (bupivacaína) a dosis crecientes hasta conseguir un alivio del dolor y del resto de la sintomatología. Una vez conseguido esto se mantuvo la infusión durante dos semanas. Pasado ese tiempo se detuvo la infusión y se retiró el catéter epidural esperando la respuesta del paciente; en tres niños el resultado ha sido la remisión del cuadro sin que hayan vuelto a aparecer los síntomas característicos del SDRC, por el contrario en los otros dos casos el dolor y el resto de la sintomatología reapareció al poco tiempo de finalizar la infusión epidural (39-41).

La decisión en estos dos niños fue la de implantar (en quirófano y bajo anestesia general) un electrodo octopolar en cordones medulares posteriores, previa información y obtención del consentimiento de los padres. El electrodo se situó a nivel de las metámeras T8-T10. Una vez que las niñas se recuperaron de la anestesia se inició la estimulación eléctrica obteniéndose un buen nivel de parestesias con remisión del dolor y del resto de los síntomas acompañantes (42). La estimulación eléctrica medular es una técnica invasiva cuya utilización puede recomendarse en aquellos casos se haya mostrado insuficiente la aplicación del resto del algoritmo de tratamiento (43).

\section{CONCLUSIONES}

La rapidez en el diagnóstico es fundamental en el SDRC en niños ya que su demora conlleva el que también se retrase el inicio del tratamiento, poniendo en peligro la recupe- ración funcional de la extremidad afectada. A diferencia de lo que ocurre con los adultos, en el SDRC en niños cuando se realiza el tratamiento adecuado los niveles de recuperación llegan al $97 \%$ de los casos. Por lo tanto la primera conclusión y, posiblemente la más importante es que tanto su reconocimiento temprano como la institución de un programa de rehabilitación adecuado al paciente, son esenciales para la consecución de un buen resultado.

En la mayoría de los trabajos citados se comprueba cómo la movilización precoz de la extremidad afectada, así como un apoyo correcto del comportamiento, pueden llevar a cambios dramáticos en los signos y síntomas del SDRC. La terapia física, el restablecimiento de la función en la extremidad afectada, las medidas conductuales y la desensibilización, son todas ellas medidas útiles para aliviar el miedo al movimiento lo que va a permitir la utilización de medidas físicas en el niño.

Si bien, la mayoría de los niños responden a la combinación de tratamiento farmacológico más terapia física y medidas conductuales, hay casos en los cuales no es posible llevarlo a cabo debido a la presencia de dolor intenso asociado a alodinia severa; precisamente es en estos pacientes donde la utilización de las diversas técnicas intervencionistas aquí descritas están indicadas con el propósito de conseguir un buen resultado final.

CORRESPONDENCIA:

Manuel J. Rodríguez-López

Unidad de Tratamiento del Dolor

Hospital Regional Universitario "Carlos Haya"

Avda. Carlos Haya, s/n

29010 Málaga

e-mail: maje1946yahoo.es

\section{BIBLIOGRAFÍA}

1. Merskey H, Bogduk N. Classification of chronic pain: Description of chronic pain syndromes and definition of pain terms. Seattle, WA: IASP Press; 1994.

2. Berde CB, Lebel A. Complex regional pain syndromes in children and adolescents. Anesthesiology 2005;102:387-91.

3. Stanton-Hicks M. Plasticity of complex regional pain syndromes (CRPS) in children. Pain Medicine 2010;11:1216-23.

4. Finnis DG, Murphy PM, Brooker C, et al. Complex regional pain syndrome in child and adolescents. Eur J Pain 2006;10:767-70.

5. Kachko L, Efrat R, Ben Ami S, et al. Complex regional pain syndromes in children and adolescents. Pediatric Int 2008;50:523-7.

6. Mitchell SW. On the diseases of nerves, resulting from injuries. In: Flint A, editor. Contributions relating to the casusation and prevention of disease, and to camp diseases. New York: US Sanitary Commissionies; 1867.

7. Schiano A, Eisinger J, Acquaviva PC. Algodystrophie du rachis. En: Les Algodystrophies. Paris: Laboratoire Armour Moutagu; 1976. p. 32-5. 
8. Rodrigo MD, Perena MJ, Serrano P, et al. Síndrome de dolor regional complejo. Rev Soc Esp Dolor 2000;11:78-97.

9. Sudeck P. Uber die akute entzundliche Knochenatrophie. Archiv fur Klinische Chirurgie 1900;62:147-65.

10. Leriche R, Fontaine R. Des osteoporoses douloureuses posttraumatiques. Presse Med 1930;38:616-21.

11. Evans JA. Reflex sympathetic distrophy. Surg Gynecol Obstet 1946;82:36-43.

12. Steinbrocker $O$. The shoulder-hand syndrome in reflex dystrophy of the upper extremity. Ann Intern Med 1948;29:2252.

13. Bonica JJ. Management of pain. Philadelphia: Lea \& Febiger; 1953.

14. Merskey H. Classification of Chronic pain. Descriptions of Chronic Pain Syndromes and definitions of Pain Terms. 2nd ed. H. IASP Press, Seattle WA; 1994.

15. Finnis DG, Murphy PM, Brooker C, et al. Complex regional pain syndrome in children and adolescent. Eur J Pain 2006; 10:767-70.

16. Lee BH, Scharff L, Sethna NF, et al. Physical therapy and cognitive-behavioral treatment for complex regional pain syndromes. J Pediatr 2002;141:135-40.

17. Veldman PH, Reymen HM, Arntz IE, et al. Signs and Symptoms of reflex sympathetic dystrophy: Prospective study of 829 patients. Lancet 1993;342:1012-6.

18. Goldschneider KR. Complex regional pain syndrome in children: Asking the right question. Pain Res Manag 2012;17:386-90.

19. Juottonen K, Gockel M, Silen T, et al. Altered central sensorimotor processing in patients with complex regional pain syndrome. Pain 2002;98:315-23.

20. Maihofner C, Handwerker HO, Neundorfer B, et al. Pattern of cortical reorganisation in complex regional pain syndrome. Neurology 2003;61:1707-15.

21. Janic W, Baron R. Complex regional pain syndrome is a disease of the central nervous system. Clin Auton Res 2002;12:150-64.

22. Schurmann M, Gradl G, Andress HJ, et al. Assessment of peripherals sympathetic nervous function for diagnosing early post-traumatic complex regional pain syndrome type I. Pain 1999;80:149-59.

23. Forouzanfar T, Koke AJA, van Kleef M, et al. Treatment of complex regional pain syndrome type I. Eur J Pain 2002;6:105-122.

24. Torebjork E, Wahren L, Wallin G, et al. Noradrenaline evoked pain in neuralgia. Pain 1995;63:11-20.

25. Taylor L. Complex regional pain syndrome: Comparing adults and adolescents. Available at: www.medscape.com/ viewarticle/430537.

26. Low AK, Ward K, Wines AP. Pediatric complex regional pain syndrome. J Pediat Orthop 2007;27:567-72.
27. Fitze G. Complex regional pain in children. Unfallchirurg 2011;114:411-6.

28. Bukhalo Y, Mullin V. Presentation and treatment of complex regional pain syndrome type I in a 3 year old. Anesthesiology 2004;101:542-3.

29. Raja SN, Grabow TS. Complex regional pain syndrome I (reflex sympathetic dystropy). Anesthesiology 2002;96:1254-60.

30. McClain BD, ed. Pediatric pain: : children and complex regional pain syndrome. Available at: www.nationalpain foundation.org/MyTreatment/articles/CRPS_Children. asp

31. Hooshmand H, Hashmi H. Complex regional pain syndrome - reflex sympathetic dystrophy syndrome diagnosis and therapy. A review of 824 patients. Pain Digest 1999;9:1-24.

32. Bruehl S, Harden RN, Galer BS, et al. Complex regional pain syndrome: Are there distinct subtypes and sequencial stages of the syndrome? Pain 2002;95:119-24.

33. Sherry DD, Wallace CA, Kelley C, et al. Short and longterm outcomes of children with complex regional pain syndrome type I treated with exercise therapy. Clin J Pain 1999;15:218-23.

34. Harris ES, Schimka KE, Carlson RM. Complex regional pain syndrome of the pediatric lower extremity: A retrospective review. J Am Podiatz Med Assoc 2012;102:99-104.

35. Tan EC, Zijlstra B, Essink ML, et al. Complex regional pain syndrome type I in children. Acta Paediatr 2008;97:875-9.

36. Sherry DD. An overview of amplified musculoskeletal pain syndromes. J Rheumatol Suppl 2000;58:44-8.

37. Wilder RT. Management of pediatric patients with complex regional pain syndrome. Clin J Pain 2006;22:443-8.

38. Zernikow B, Dobe M, Hirschfeld G, et al. Please don't hurt me!: A plea against invasive procedures in children and adolescents with CRPS. Schmerz 2012;26:389-95.

39. Farid IS, Heiner EJ. Intrathecal local anesthetic infusion as a treatment for complex regional pain syndrome in a child. Anesthesia \& Analgesia 2007;104:1078-80.

40. Bredahl C, Kristensen AK, Christensen KS. Trewatment of reflex dystrophy with continuous Peripherals nerve block. Ugeskr Laeger 2007;169:59-60.

41. Meier PM, Zurakowski D, Berde CB, et al. Lumbar sympathetic blockade in children with CRPS: a double blind placebo-controlled crossover trial. Anesthesiology 2009;111:372-80.

42. Rodríguez-López MJ, Fernández-Baena M, Aldaya-Valverde C. Tratamiento del SDRC en niños mediante la estimulación eléctrica medular. Rev Soc Esp Dolor 2013;20:180-5.

43. Olsson GL, Meyerson BA, Linderoth B. Spinal cord stimulation in adolescents with complex regional pain syndrome type I. Eur J Pain 2008;12:53-9. 